\title{
Квантовый размерный эффект и осцилляции Шубникова-де Гааза в поперечном магнитном поле в полупроводниковых нитях $\mathrm{Bi}_{0,92} \mathrm{Sb}_{0,08}$
}

\author{
А. А. Николаева ${ }^{a^{*}}$, Л. А. Конопко, Т. Е. Хубер ${ }^{b^{* *}}$, \\ И. А. Попов ${ }^{\text {a }, ~ Г . ~ И . ~ П а р а ~}{ }^{\text {a }, ~ О . ~ В . ~ Б о т н а р ь ~}{ }^{\text {a }}$ \\ ${ }^{a}$ Институт электронной инженерии и нанотехнологий им. Д. Гииу, \\ 2. Китинев, МD-2028, Молдова \\ ${ }^{b}$ Гарвардский университет, Вашингтон, США \\ e-mail:A.Nikolaeva@nanotech.md \\ e-mail: titoehuber@gmail.com \\ Поступила в редакцию 17.06.2021 \\ После доработки 15.09.2021 \\ Принята к публикации 17.09.2021
}

\begin{abstract}
Исследованы транспортные свойства, магнетосопротивление и осцилляции Шубникова-де Гааза (ШдГ) монокристаллических нитей $\mathrm{Bi}_{0,92} \mathrm{Sb}_{0,08}$ с диаметрами от 180 нм до 2,2 мкм, полученных литьем из жидкой фазы в стеклянной оболочке с ориентацией (1011) вдоль оси нити. Впервые обнаружено, что энергетическая щель $\Delta E$ возрастает в 4 раза с уменьшением диаметра нитей $d$ за счет проявления квантового размерного эффекта. Значительное возрастание энергетической щели может происходить в условиях линейного закона дисперсии энергии от импульса, характерного как для бесщелевого состояния, так и для поверхностных состояний топологического изолятора. Показано, что в сильном магнитном поле при низких температурах происходит переход полупроводник-полуметалл, проявляющийся в температурных зависимостях сопротивления. Анализ осцилляций ШдГ-сдвиг фазы уровней Ландау, особенности угловых зависимостей периодов осцилляций указывают, что совокупность проявления свойств топологического изолятора и квантового размерного эффекта приводит к проявлению новых эффектов в размерно-ограниченных структурах, требующих иных научных подходов и применений в микроэлектронике.
\end{abstract}

Ключевые слова: квантовый размерный эффект, топологический изолятор, поверхностные состояния, монокристаллические нанонити, квантовые осцилляции, поперечное магнетосопротивление

УДК 537.9, 53.06, 53.043

https://doi.org/10.52577/eom.2021.57.6.79

\section{ВВЕДЕНИЕ}

Сплавы $\mathrm{Bi}_{1-\mathrm{x}} \mathrm{Sb}_{\mathrm{x}}$ относятся к системе полупроводников с инверсией зон типа полуметаллполупроводник. С изменением концентрации сурьмы от нуля до единицы энергетический спектр висмута непрерывно перестраивается в спектр сурьмы [1-3].

Общая схема перестройки энергетического спектра сплавов $\mathrm{Bi}_{1-\mathrm{x}} \mathrm{Sb}_{\mathrm{x}}$ в интервале $(0,05<x<0,22)$ приведена на рис. 1 [4].

Согласно анализу многочисленных исследований, проведенных в работе [4], наиболее достоверными являются следующие результаты: энергетический спектр в висмуте - инверсионный с $E g_{L}=-9$ мэВ, энергия Ферми электронов $E_{F L}=31$ мэВ, энергия Ферми дырок $E_{F T}=12,2$ мэB, инверсия термов Ls и La имеет место при $x=0,04 \mathrm{Sb}$.

Переход полуметалл-полупроводник с косвенной щелью между La и T происходит при $x=0,07 \mathrm{Sb}$, максимальное значение прямой щели в полупроводниковом сплаве $E g_{L}=25$ мэВ наблюдается при $x=0,15$, переход из полупроводникового состояния в металлическое совершается при $x=0,22$.

Неугасающий интерес к сплавам $\mathrm{Bi}_{1-\mathrm{x}} \mathrm{Sb}_{\mathrm{x}}$ обусловлен тем, что, с одной стороны, в размерно-ограниченных структурах на их основе эффект размерного квантования реализуется при достаточно больших толщинах -100-500 нм [5], и на таких структурах предсказывалось значительное возрастание термоэлектрической эффективности [6]. Для наблюдения эффектов размерного квантования необходимы тонкие монокристаллические образцы. $\mathrm{C}$ другой стороны, сплавы $\mathrm{Bi}_{1-\mathrm{x}} \mathrm{Sb}_{\mathrm{x}}(0,05<x<0,22)$ в полупроводниковой области концентраций относятся к новому классу материалов, открытых сравнительно недавно, топологическим изоляторам (ТИ), которые являются электропроводными только на поверхности [7-9]. Впервые поверхностные состояния внутри объемной запрещенной зоны с линейным законом дисперсии экспериментально наблюдались в полупроводниковых сплавах 


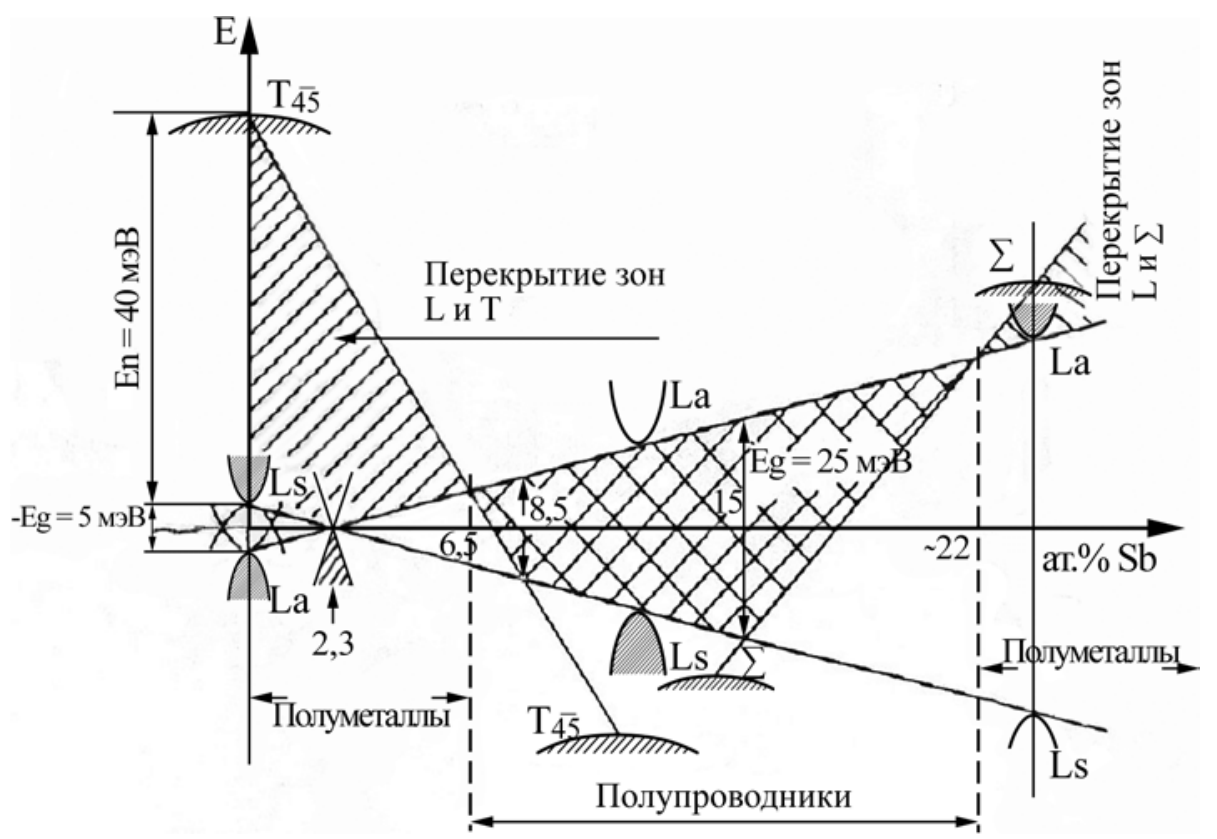

Рис. 1. Схема перестройки зонной структуры $\mathrm{Bi}_{1-\mathrm{x}} \mathrm{Sb}_{\mathrm{x}}$ в зависимости от $x(0 \leq x \leq 0,25)$ при $4,2 \mathrm{~K}$.

$\mathrm{Bi}_{1-\mathrm{x}} \mathrm{Sb}_{\mathrm{x}} \mathrm{c}$ помощью фотоэмиссионной спектроскопии с угловым разрешением (APFRS) [10].

В работе [11] было показано, что разные вариации конуса Дирака в электронной зонной структуре с различной анизотропией $2 \mathrm{D}$ могут быть сконструированы в тонких пленках $\mathrm{Bi}_{1-\mathrm{x}} \mathrm{Sb}_{\mathrm{x}}$. Дираковские конусы, наблюдаемые в топологических изоляторах, идентифицируются как новый класс материалов в качестве кандидатов для квантовых компьютеров в спинтронике, многообещающего будущего термоэлектричества $[5,6,8]$.

В настоящей работе приведены результаты исследования толщинных и температурных зависимостей сопротивления, магнетосопротивления, осцилляций Шубникова-де Гааза в перпендикулярном магнитном поле монокристаллических полупроводниковых нитей $\mathrm{Bi}_{0,92} \mathrm{Sb}_{0,08}$ различных диаметров, полученных литьем из жидкой фазы в стеклянной оболочке.

\section{МЕТОДИКА ЭКСПЕРИМЕНТА}

Монокристаллические нити состава $\mathrm{Bi}_{0,92} \mathrm{Sb}_{0,08}$ различных диаметров в стеклянной изоляции получались литьем из жидкой фазы по методу Улитовского [12-14].

Предварительно монокристаллы сплава $\mathrm{Bi}_{0,92} \mathrm{Sb}_{0,08}$ изготавливались методом зонной перекристаллизации.

Кристаллизация жилы микропровода в стеклянной оболочке протекает при сильном переохлаждении на фоне перекристаллизации. Сильное переохлаждение и высокие скорости перекристаллизации способствуют росту монокристаллической жилы и сохранению стехиометрического состава $\mathrm{Bi}_{1-\mathrm{x}} \mathrm{Sb}_{\mathrm{x}}$ на всей длине. Все образцы были строго цилиндрической формы, в сечении - окружность, что подтверждено снимками в сканирующем электронном микроскопе.

Монокристалличность и кристаллографическая ориентация нитей $\mathrm{Bi}_{0,92} \mathrm{Sb}_{0,08}$ были установлены с помощью рентгенографических исследований (X-Ray diffraction рентгенограммы вращения). Как и нити чистого $\mathrm{Bi}$ и $\mathrm{Bi}-17 \mathrm{aT} \% \mathrm{Sb}$ [14-16], изготовленные аналогичным способом, нити $\mathrm{Bi}_{0,92} \mathrm{Sb}_{0,08}$ были монокристаллическими, имели одну и ту же ориентацию (1011) вдоль оси нити, при которой биссекторная ось $C_{1}$ отклонена на $20^{\circ}$ от оси нити, а бинарная ось $C_{2}$ строго перпендикулярна оси нити (рис. 2).

При такой кристаллографической ориентации нитей направление вытянутости одного электронного эллипсоида образует угол $\approx 20^{\circ} \mathrm{c}$ осью нити, а его сечение $\mathrm{S}_{1}$ с плоскостью, перпендикулярной оси нити, близко к минимальному, а два других $S_{2,3}$ расположены симметрично относительно оси нити, их сечение с плоскостью, перпендикулярной оси нити, $S_{2,3}=2 S_{1}[12-14]$.

Измерения проводились двухконтактным методом с использованием InGa эвтектики. Омичность контактов проверялась снятием вольт-амперных характеристик в интервале температур 4,2-300 К. Исследования магнетополевых зависимостей и осцилляций Шубникова-де Гааза в перпендикулярном магнитном поле $(H \perp I)$ проводились в поле сверхпроводящего соленоида в магнитных полях до $14 T$, при температурах 4,2-300 K. 


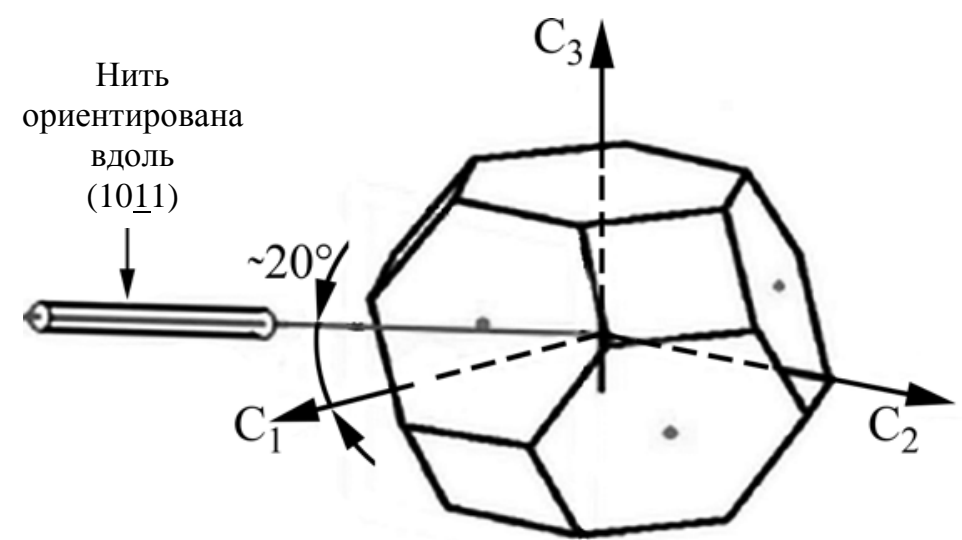

Рис. 2. Приведенная зона Бриллюэна Ві в гексагональной упаковке и ориентации (1011) нитей $\mathrm{Bi}_{1-\mathrm{x}} \mathrm{Sb}_{\mathrm{x}}$, полученных методом Улитовского.
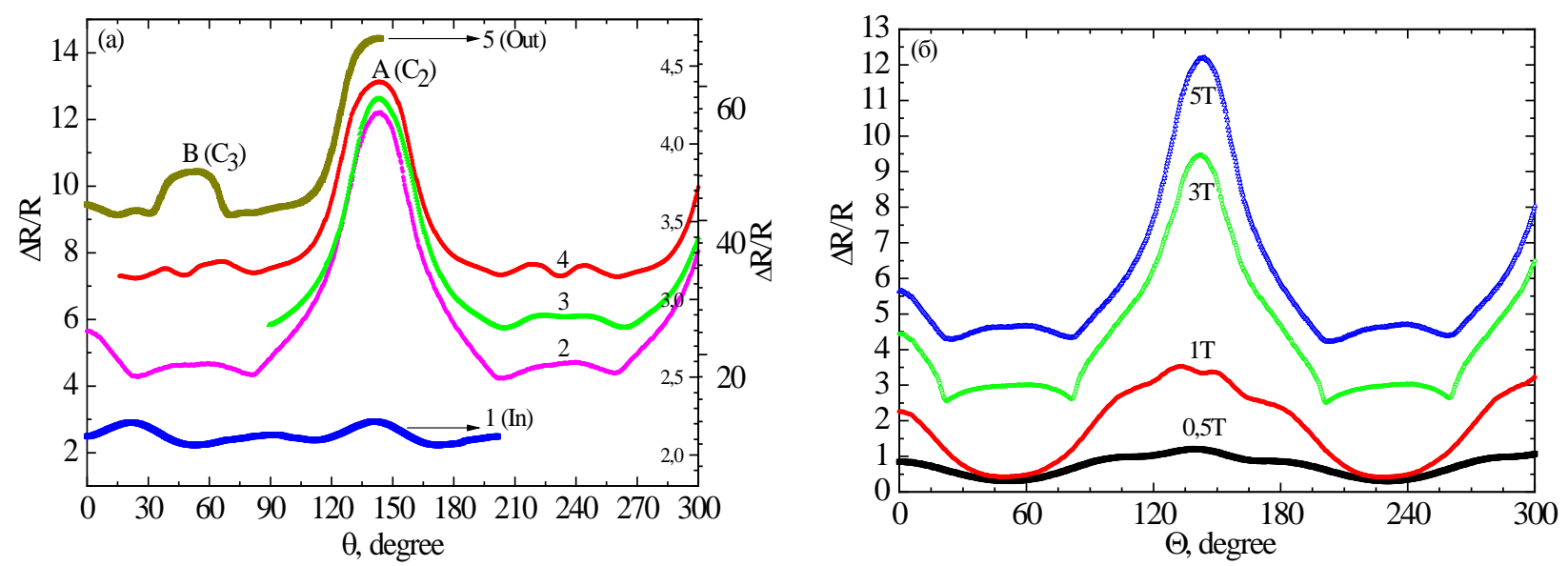

Рис. 3. Диаграммы вращения поперечного магнетосопротивления: (а) - $(H \perp I)$ нитей $\mathrm{Bi}_{0,92} \mathrm{Sb}_{0,08}$ при $4,2 \mathrm{~K}, H_{\perp}=14 T$ : $1-d=0,18 \mu \mathrm{m}$ (ось $Y$, шкала справа внутри); $2-d=0,39 \mu \mathrm{m} ; 3-d=0,5 \mu \mathrm{m} ; 4-d=0,55 \mu \mathrm{m}$ (шкала слева); $5-d=2,2 \mu \mathrm{m}$ (ось $Y$, шкала справа снаружи); (б) $-\Delta R / R(\theta)$ нити $\mathrm{Bi}_{0,92} \mathrm{Sb}_{0,08}$ при $4,2 \mathrm{~K}, d=0,5 \mu \mathrm{m}$, при различных значениях магнитного поля $H$.

При исследованиях осцилляций Шубниковаде Гааза (ШдГ) использовалась стандартная модуляционная техника, позволяющая записывать производную сигналов $\partial R / \partial H(H)$ в интервале температур 1,5-10 K, при произвольной ориентации магнитного поля относительно кристаллографических осей исследуемых нитей. Удельное сопротивление $\rho$ при $300 \mathrm{~K}$ рассчитывалось с использованием соотношения $\rho=R \frac{\pi d^{2}}{4 l}$, где $l$ - длина нити; $d$ - диаметр.

Диаграммы вращения поперечного магнетосопротивления (H $\quad \perp \quad I)$ снимались как для подтверждения монокристалличности исследуемых нитей, так и для установки направления магнитного поля вдоль определенной кристаллографической оси нити.

\section{РЕЗУЛЬТАТЫ И ОБСУЖДЕНИЕ}

На рис. 3 представлены диаграммы вращения поперечного магнетосопротивления (ДВПМ) нитей $\mathrm{Bi}_{0,92} \mathrm{Sb}_{0,08}$ различных диаметров при температуре $4,2 \mathrm{~K}$ в магнитном поле $H=14 T$ $(H \perp I)$, а также ДВПМ при различных значениях магнитного поля.
Точка $A$ соответствует $H \| C_{2}$, точка $B\left(H \perp C_{2}\right)$ направлению, отклоненному от биссекторной оси на $20^{\circ}$ в биссекторнотригональной плоскости (рис. 2).

Структура ДВПМ нитей идентична для нитей всех диаметров и близка к аналогичным диаграммам вращения поперечного магнетосопротивления массивных монокристаллических образцов с биссекторной осью вдоль длины образца и перпендикулярной магнитному полю, то есть при вращении магнитного поля в бинарно-тригональной плоскости [17]. Это является подтверждением одной и той же кристаллографической ориентации исследуемых нитей. При уменьшении диаметра нитей $d<0,55 \mu \mathrm{m}$ анизотропия магнетосопротивления $R_{\max } / R_{\min }$ уменьшается. Наиболее существенно анизотропия магнетосопротивления зависит от диаметра нитей $d$ при гелиевых температурах.

Ослабление эффективности магнитного поля с уменьшением диаметра нитей $d$ свидетельствует об уменьшении подвижностей носителей заряда, связанных с влиянием поверхностного рассеяния, усиливающегося с уменьшением диаметра. Выравнивание подвижностей носителей заряда в условиях проявления класси- 


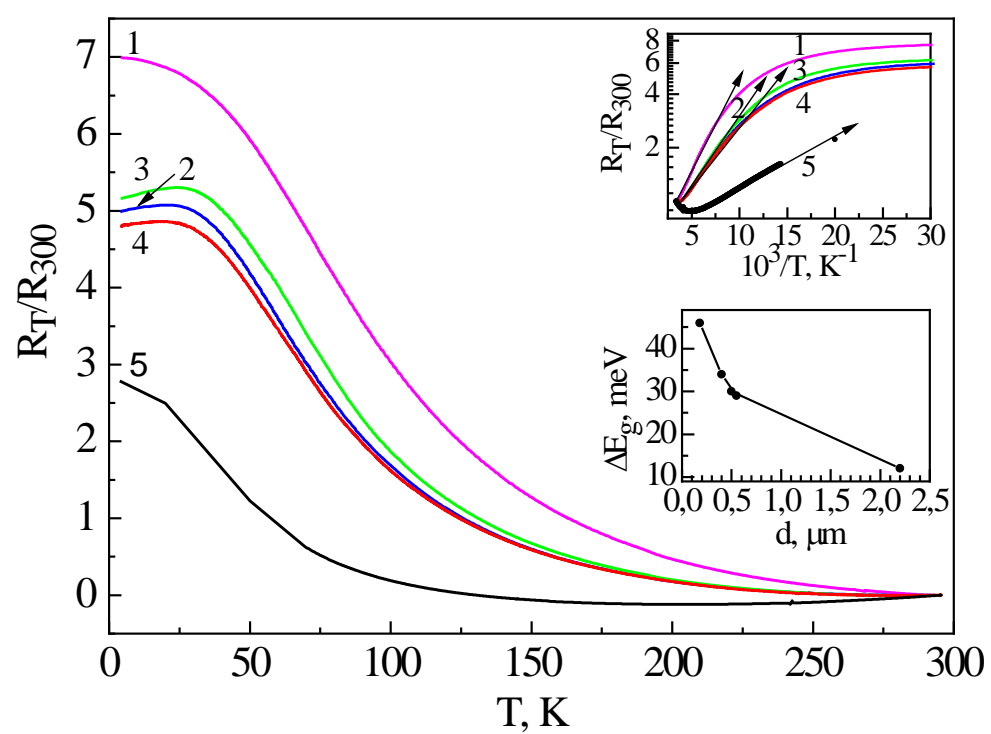

Рис. 4. Температурные зависимости относительного сопротивления $R_{T} / R_{300}(T)$ для нитей $\mathrm{Bi}_{0,92} \mathrm{Sb}_{0,08}$ с диаметрами: $1-d=0,18 \mu \mathrm{m} ; 2-d=0,39 \mu \mathrm{m} ; 3-d=0,5 \mu \mathrm{m} ; 4-d=0,55 \mu \mathrm{m} ; 5-d=2,2 \mu \mathrm{m}$. На вставке вверху - зависимость $\ln \rho\left(T^{-1}\right)$, на вставке внизу - зависимость энергетической щели $\Delta E$ от диаметра нитей $d$.

ческого размерного эффекта происходит вследствие того, что поверхностное рассеяние сильнее влияет на высокоподвижные носители, чем малоподвижные.

На рис. 4 представлены температурные зависимости приведенного сопротивления $R_{T} / R_{300}(T)$ нитей $\mathrm{Bi}_{0,92} \mathrm{Sb}_{0,08}$ различных диаметров в интервале температур 4,2-300 K.

При $300 \mathrm{~K}$ удельное сопротивление практически не зависит от диаметра нитей $d$ и совпадает с удельным сопротивлением массивных монокристаллов $\rho_{x}=145 \times 10^{-6} \mathrm{Oм} \cdot \mathrm{cm}$ [17].

На зависимостях $\ln \rho(1 / T)$ проявляются четкие экспоненциальные участки $\rho_{\mathrm{T}}=\rho_{0}{ }^{*} \exp \left(\Delta E / 2 k_{0} T\right)$, наклон которых и область существования зависят от диаметра нитей $d$ (рис. 4, вставка вверху). Для нитей с $d \approx 2,2$ мкм величина $\Delta E$, рассчитанная из анализа зависимостей $\ln \rho\left(T^{-1}\right)$ (кривая 5), составляла 11-12 мэВ, что хорошо согласуется со значением для массивных образцов аналогичного состава. Как было показано в работах [3, 4], в полупроводниковых сплавах $\mathrm{Bi}_{1-\mathrm{x}} \mathrm{Sb}_{\mathrm{x}}$ с $0,07<x<0,09$ минимальной энергетической щелью является непрямая щель $\Delta E_{L T}$ (рис. 1), равная расстоянию между дном зоны проводимости в точке $L$ зоны Бриллюэна и потолком валентной зоны в точке $T$. При уменьшении диаметра нитей $d$ энергетическая щель возрастает, что связано с проявлением квантового размерного эффекта [5].

Впервые было обнаружено, что с уменьшением диаметра нити $d$ энергетическая щель $\Delta E$ возрастала почти в 4 раза и достигала значения 47 мэВ в нитях с $d \approx 180$ нм. Отметим, что в аналогичных нитях состава $\mathrm{Bi}-17 \mathrm{aT} \% \mathrm{Sb}$ энергетическая щель $\Delta E_{g}$ возрастала в $\approx 2$ раза при значительно меньших диаметрах нитей $(d=75$ нм $)[14,15]$.

Зависимость энергетической щели $\Delta E \sim d^{-1}$ (рис. 4 , вставка) указывает на то, что причиной возрастания $\Delta E$ при уменьшении диаметра нитей $d$ является квантовый размерный эффект (КРЭ), предсказанный в работах $[5,6]$ и наблюдаемый в нитях $\mathrm{Bi}-17 \mathrm{aT} \% \mathrm{Sb}$ [15].

Такое значительное возрастание энергетической щели может быть связано с тем, что, как было показано в [7, 10], вблизи бесщелевого состояния электроны ведут себя как безмассовые частицы, у которых закон дисперсии отличается от квадратичного $E=p^{2} / 2 m$ и становится близок к закону драковского типа, при котором имеет место линейная зависимость энергии от импульса $E=h k v_{F}$, где $v_{F}-$ фермиевская скорость электронов. Это приводит к более существенному смещению энергетических зон за счет квантового размерного эффекта и возрастанию щели до 47 мэВ.

Именно поэтому в отличие от нитей состава $\mathrm{Bi}-17 \mathrm{aT} \% \mathrm{Sb}$ поверхностные состояния, с законом дисперсии дираковского типа, характерные для топологических изоляторов, не приводят к уменьшению сопротивления и металлической проводимости при температуре $T<50 \mathrm{~K}[14,15]$.

\section{МАГНЕТОСОПРОТИВЛЕНИЕ}

Исследовалось поперечное магнетосопротивление $R(H)(H \perp I)$ в точках $A$ и $B$ (рис. 3) в магнитных полях до $14 T$ при различных темпеpaтурах. На рис. 5 приведены полевые зависимости магнетосопротивления $R_{H} / R_{0}(H)$ в точках $A$ и $B$, соответствующие $H \| C_{2}$ и $H \| C_{3}$ на диаграммах вращения поперечного магнетосопротивления (рис. 3) в магнитных полях до 

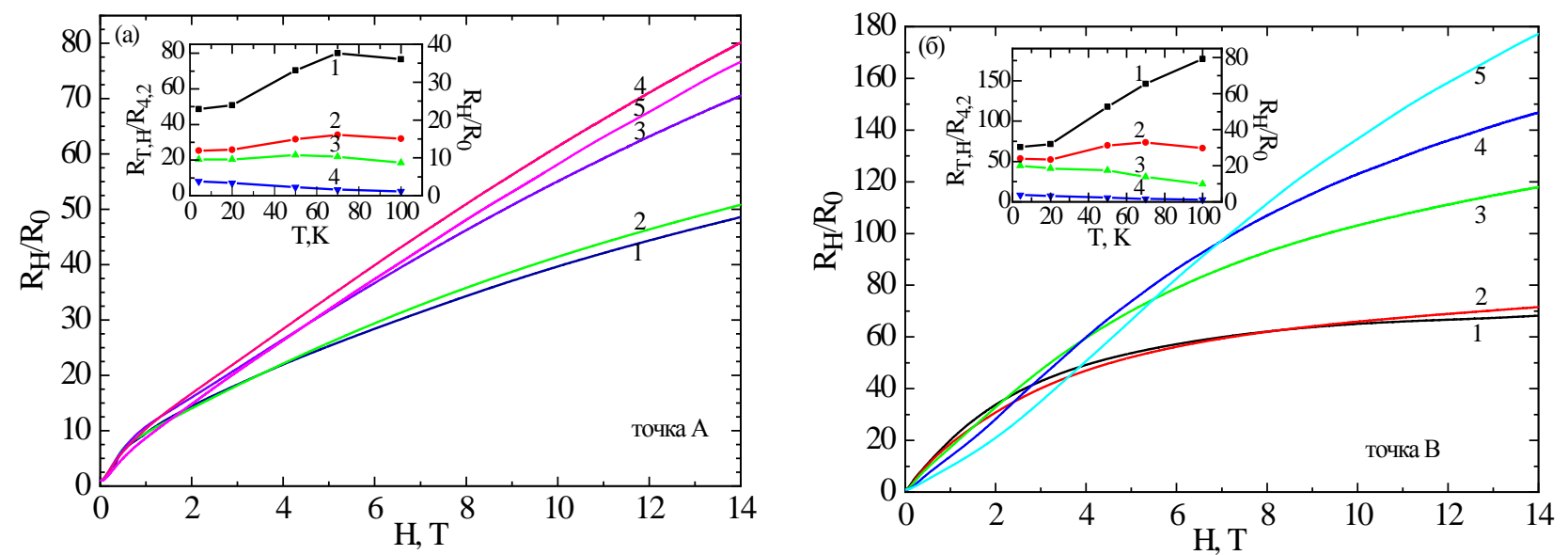

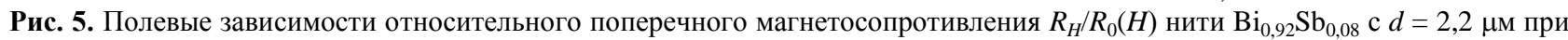
различных температурах: (а) $-H \| C_{2}$, (б) $-H \| C_{3}$ (точки $A$ и $B$ на угловой диаграмме вращения). На вставках: температурные зависимости сопротивления в магнитном поле $R_{T, H} / R_{4 / 2}(T): 1-H=14 T$; $2-H=5 T$; $3-H=1 T ; 4-H=0$, (кривые 3, 4 -шкала справа)
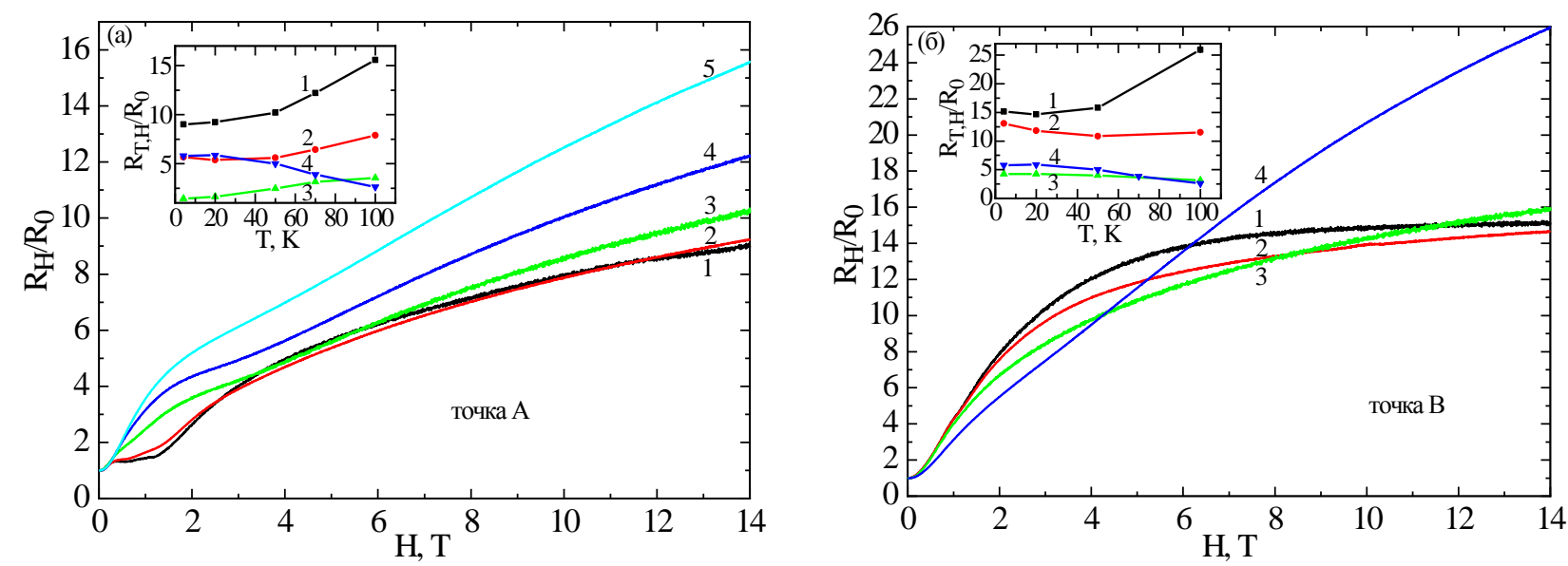

Рис. 6. Полевые зависимости относительного поперечного магнетосопротивления $R_{H} / R_{0}(H)$ нити $\mathrm{Bi}_{0,92} \mathrm{Sb}_{0,08} \mathrm{c} d=0,55 \mu$ м при различных температурах: (а) $-H \| C_{2}$, (б) $-H \| C_{3}$. Вставки: температурные зависимости сопротивления в магнитном поле $R_{T, H} / R_{4 / 2}(T)$ при: $1-H=14 T ; 2-H=5 T ; 3-H=1 T ; 4-H=0$.

$14 T$ при различных температурах $1-T=4,2 \mathrm{~K}$; $2-T=20 \mathrm{~K} ; 3-T=54 \mathrm{~K} ; 4-T=72 \mathrm{~K}$; $5-T=100 \mathrm{~K}$.

В слабых магнитных полях $(H<2$ T) характерен монотонный рост магнетосопротивления (МС) с увеличением магнитного поля при $H \| C_{2}$ и при $H \| C_{3}$, при этом относительное магнетосопротивление $R_{H} / R_{0} \mathrm{MC}$ при $4,2 \mathrm{~K}$ больше, чем при $100 \mathrm{~K}$. Однако, в сильных магнитных полях $(H>3 T)$ характер кривых качественно меняется. Относительное магнетосопротивление $R_{14 T} / R_{0}(H)$ при $4,2 \mathrm{~K}$ становится меньше, чем при $100 \mathrm{~K}$, а при $H \| C_{3}$ стремится к насыщению в магнитных полях $>3 T$ (рис. 5, 6(б), кривые 1, 2), так как подвижность носителей тока в магнитном поле уменьшается.

Насыщение и уменьшение сопротивления $R_{14 T} / R_{0}(H)$ в сильных магнитных полях при низких температурах в полупроводниковых нитях $\mathrm{Bi}_{1-\mathrm{x}} \mathrm{Sb}_{\mathrm{x}}$ могут наблюдаться только при такой перестройке энергетического спектра в квантующем магнитном поле, при котором

происходит увеличение концентрации носителей заряда.

Возрастание концентрации электронов и дырок у нитей $\mathrm{Bi}_{0,92} \mathrm{Sb}_{0,08}$ в сильных магнитных полях при $H \quad \| \quad C_{3}$ является следствием электронного перехода полупроводникполуметалл, индуцированного сильным магнитным полем и обусловленного возникновением перекрытия $L$-электронного и $T$-дырочного-экстремумов [18].

При низкой температуре в направлении квантующего магнитного поля вдоль $C_{3}$, происходит сближение $L$-термов, a дырочный экстремум в точке $T$ импульсного пространства поднимается по шкале энергий вверх, в результате чего прямая $\Delta E_{L}$ и косвенная $\Delta E_{L T}$ щели уменьшаются и могут перекрываться.

На вставках рис. 5, 6 приведены температурные зависимости $R_{T, H} / R_{4,2}(T)$ (кривые $1-3$ ) в магнитном поле и $R_{T, H} / R_{4,2}(T)$ без магнитного поля (кривая 4) в области температур 4,2-100 K для нитей $\mathrm{Bi}_{0,92} \mathrm{Sb}_{0,08}$ двух различных диаметров $d=2,2$ мкм и $d=0,55$ мкм в точках $A$ и $\bar{E}$ 


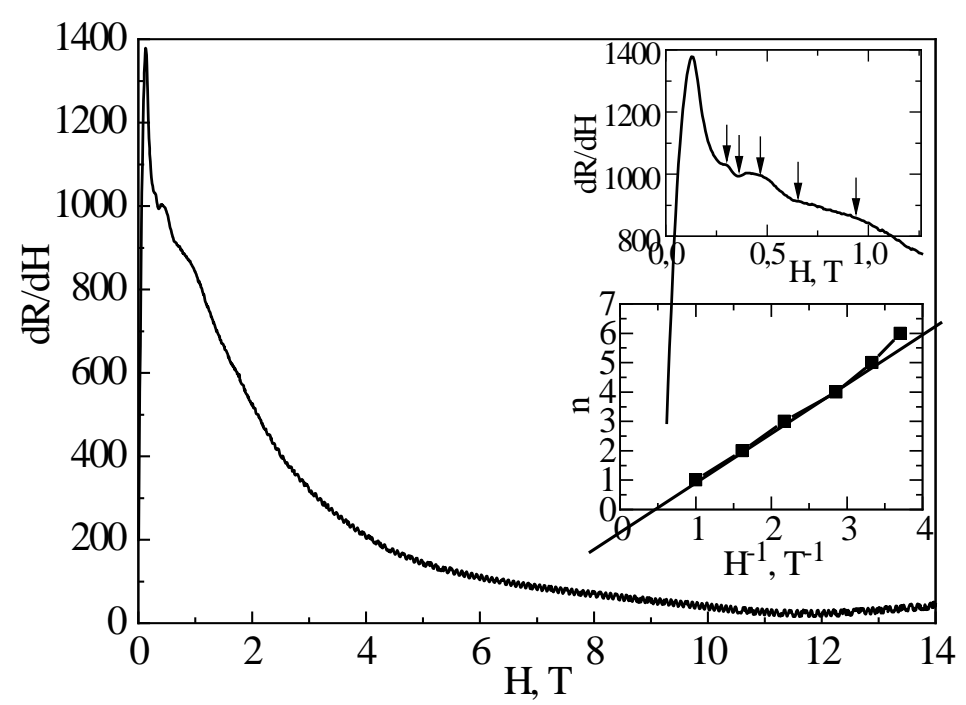

Рис. 7. Осцилляции Шубникова-де Гааза на зависимостях $\partial R / \partial H(H)$ на поперечном магнетосопротивлении при $H \| C_{3}$ (точка $B$ на диаграмме вращения). На вставке: начальный участок кривой (вверху) и зависимость квантового номера $n$ осцилляций ШдГ от обратного магнитного поля $H^{1}$ (внизу).
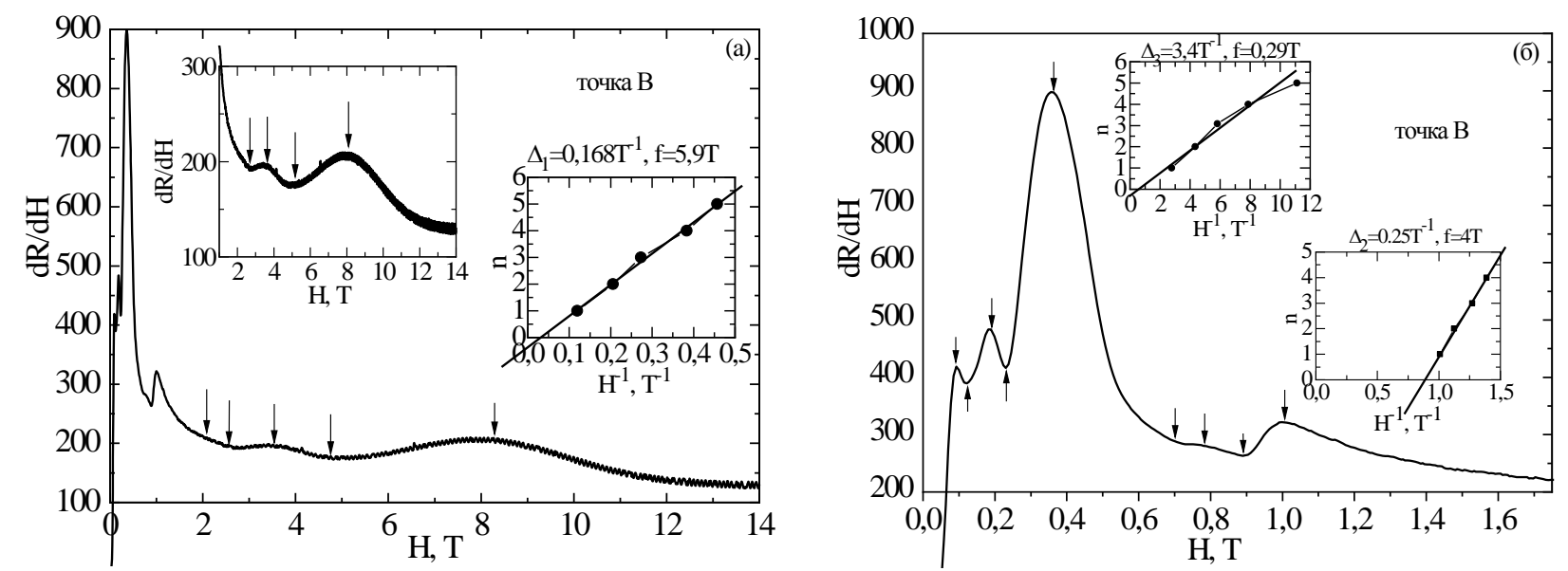

Рис. 8. Осцилляции Шубникова-де Гааза на зависимостях $\partial R / \partial H(H)$ поперечного магнетосопротивления (точка $A$ на диаграмме вращения). На вставках (а) $\partial R / \partial H(H)$ в области магнитных полей $>2 T$ (вверху) и зависимость квантового номера $n$ осцилляций ШдГ от обратного поля $H^{-1}$; (б) начальный участок кривой $\partial R / \partial H(H)$ и соответствующие зависимости $n\left(H^{-1}\right)$ (вставки).

(на диаграммах вращения). Было обнаружено, что в сильном магнитном поле $(H=14 T)$ температурные зависимости относительного сопротивления $R_{T} / R_{4,2}(T) \quad$ имеют «металлический» характер (кривые 1, 2 на вставках). Сопротивление при $T<100 \mathrm{~K}$ уменьшается в 2,6 раза в нитях с $d=2,2 \mu \mathrm{M}$, у которых щель минимальна $(\approx 11$ мэВ), в то время как в отсутствие магнитного поля зависимость $R(T)$ имеет полупроводниковый вид (кривые 4 на вставках). У более тонких нитей эффект перехода полупроводник-полуметалл проявлялся слабее (кривые $1-2)$.

Аналогичный эффект в сильном магнитном поле в полупроводниковых сплавах наблюдался в массивных образцах сплавов $\mathrm{Bi}_{1-\mathrm{x}} \mathrm{Sb}_{\mathrm{x}}$, легированных Те [18].

\section{ОСЦИЛЛЯЦИИ ШУБНИКОВА-ДЕ ГААЗА}

На рис. 7, 8 приведены экспериментальные зависимости производных ПМС $\partial R / \partial H(H)$ при

4,2 K в магнитных полях до $14 T$ при $H \| C_{3}$ (рис. 7) и $H \perp C_{3}$ (рис. 8) нити $\mathrm{Bi}_{0,92} \mathrm{Sb}_{0,08} \mathrm{c}$ $d=2,2 \mu \mathrm{M}$.

В случае $H \| C_{3}$ на зависимости $\partial R / \partial H(H)$ наблюдались осцилляции ШдГ только с одним периодом, в слабых магнитных полях $(H<1 T)$ (рис. 7, вставка вверху). Период осцилляций, определяемый из зависимости квантового номера $n$ ШдГ осцилляций от обратного магнитного поля $n\left(H^{1}\right)$ составляет $\Delta=1,25 T^{1}$, то есть частота ШдГ осцилляций $f=1 / \Delta=0,8 T$.

При $H \perp C_{3}$ (рис. 8) наблюдается наличие трех областей квантовых осцилляций ШдГ, эквидистантных по обратному магнитному полю $H^{1} \mathrm{c}$ тремя периодами. Низкочастотные осцилляции проявляются в слабом магнитном поле $(H<0,5 T)$ (рис. 8б) высокочастотные в сильных магнитных полях (рис. 8а, вставка) $1,5<H<14 T$ и в промежуточной области магнитных полей 0,5 $T<H<1,2 T$ (рис. 8б, вставка). 
На рис. 8а,б (вставки) приведены зависимости квантового номера $n$ осцилляций ШдГ от обратного магнитного поля $H^{1}$. Согласно расчетам, периоды квантовых осцилляций составляют в слабом магнитном поле $(H<0,5 T)$ $\Delta_{1}=3,4 T^{1}$, в промежуточной области $\Delta_{2}=0,25 T^{-1}$ и в сильном магнитном поле $(1,5<H<14 T) \Delta_{3}=0,17 T^{-1}$. Соответствующие им частоты $f=1 / \Delta: f_{1}=0,29 T, f_{2}=4 T, f_{3}=5,9 T$. При этом величина квантового предела $H_{\text {к.п. }}$ магнитного поля не совпадает ни с одной частотой ШдГ осцилляций.

Качественно результаты совпадают с данными, полученными на массивных образцах ТИ состава $\mathrm{Bi}_{0,91} \mathrm{Sb}_{0,09}$ из осцилляций де Гааза-ван Альфена $[17,19]$, - одна частота ШдГ осцилляций при $H \| C_{3}$ и три частоты при $H \| C_{2}$. Однако величины периодов ШдГ осцилляций в нитях превышают значения, полученные в массивных образцах, что, вероятно, связано со спецификой проявления квантового размерного эффекта в $1 D$ системах и проявлялось в полупроводниковых пленках $\mathrm{Bi}_{1-\mathrm{x}} \mathrm{Sb}_{\mathrm{x}}$ [11].

Как и в массивных образцах в случае $H \| C_{3}$, так и при $H \| C_{2}$ зависимость квантового номера $n\left(H^{-1}\right)$ ШдГ осцилляций имеет особенность в виде фазового сдвига $\gamma=0,5 T^{1}$ (вставки на рис. 7,8$)$. Известно, что уровни Ландау характеризуются индексом $\mathrm{n}$ и фазовым сдвигом $\gamma$, которые определяются выражением: $2 \pi(n, \gamma)=S\left(K_{F}\right) h / e H[20,21]$.

Экспериментальное значение фазового сдвига индекса $\gamma$ уровней Ландау определяется величиной $n$ для предельного значения магнитного поля при $H^{-1}=0$ в результате линейной экстраполяции зависимости экспериментальных значений $n$ от обратного магнитного поля $H^{-1}=0$. Известно, что фазовый сдвиг связан с фазой Берри, которая является интегральной характеристикой кривизны циклотронной орбиты и дисперсии электронов, и $\gamma=0,5$ для поверхностных дираковских фермионов с линейной дисперсией, описывающих поверхностные состояния.

Таким образом, осцилляции ШДГ, наблюдаемые в широкой области магнитных полей в полупроводниковых нитях ТИ $\mathrm{Bi}_{1-x} \mathrm{Sb}_{\mathrm{x}}$, являются новым эффектом, отражающим специфику проявления свойств ТИ в $1 \mathrm{D}$ системах, которые ведут к проявлению новой физики в транспортных свойствах ТИ в размерно-ограниченных структурах.

\section{ВЫВОДЫ}

Монокристаллические нити на базе сплавов $\mathrm{Bi}_{1-x} \mathrm{Sb}_{\mathrm{x}}$ в стеклянной оболочке, полученные литьем из жидкой фазы различных диаметров, являются надежным объектом для изучения квантового транспорта и особенностей проявления свойств топологических изоляторов в 1D системах.

В работе приведены результаты экспериментального исследования температурных зависимостей сопротивления, магнетосопротивления, осцилляций Шубникова-де Гааза в монокристаллических, полупроводниковых нитях $\mathrm{Bi}_{1-0,92} \mathrm{Sb}_{0,08}$ различных диаметров. Из температурных зависимостей сопротивления установлено, что энергетическая щель возрастает с уменьшением диаметра нитей в 4 раза за счет проявления квантового размерного эффекта. В сильных магнитных полях наблюдался переход полупроводник-полуметалл, индуцированный сильным магнитным полем. Анализ угловых зависимостей осцилляций ШдГ, сдвиг фазы уровней Ландау, характерный для ТИ, аномальный рост энергетической щели с уменьшением диаметра нитей указывают на возможность проявления новых эффектов ТИ в размерно-ограниченных 1D структурах, не свойственных другим системам.

\section{ФИНАНСИРОВАНИЕ РАБОТЫ}

Данная работа выполнена благодаря финансовой поддержке Государственной программы Министерства образования, культуры и инноваций Молдовы (проект №20.80009.5007.02) и американских фондов: NSF through STC CIQM 1231319, the Boeing Company and the Keck Foundation.

\section{КОНФЛИКТ ИНТЕРЕСОВ}

Авторы заявляют, что у них нет конфликта интересов.

\section{ЛИТЕРАТУРА}

1. Lerner, L.S., Cuff, F. and Williams, L.N., Energy-band parameters and relative motions in the $\mathrm{Bi}-\mathrm{Sb}$ alloy system near the semimetal-semiconductor transition, Rev. Mod. Phys., 1968, vol. 40, no. 4, p. 770.

2. Golin, S., Band model for bismuth-antimony alloy, Phys. Rev., 1968, vol. 176, no. 3, p. 830.

3. Brandt, N.B., Semenov, M.V. and Falkovsky, L.A., Experiment and theory on the magnetic susceptibility of Bi-Sb alloys, J. Low Temp. Phys., 1977, vol. 27, p. 75.

4. Миронова, Г.А., Судакова, М.В., Пономарев, Я.Г., Исследование зонной структуры полупроводниковых сплавов $\mathrm{Bi}_{1-x} \mathrm{Sb}_{x}$, ЖЭТФ, 1980, т. 76, № 5, c. 1832.

5. Tang, S. and Dresselhaus, M.S., Electronic phases, band gaps, and band overlaps of bismuth antimony nanowires, Phys. Rev. B., 2014, vol. 89, no. 4, art. ID: 045424. doi: 10.1103/PhysRevB.89.045424 
6. Cornett, J.E. and Oded, R., Thermoelectric figure of merit calculations for semiconducting nanowires, Appl. Phys. Lett., 2011, vol. 98, no. 18, art. ID: 182104. https://doi.org/10.1063/1.3585659

7. Fu, L. and Kane, C., Topological insulators with inversion symmetry, Phys. Rev. B., 2007, vol. 76, no. 4, art. $\quad$ ID: 045302. https://doi.org/10.1103/PhysRevB.76.045302

8. Qi, X.L., Zang L.R. and Zhang, S.C., Inducing a Magnetic Monopole with Topological Surface States, Science, 2009, vol. 323, no. 5918, p. 1184 doi: $\underline{10.1126 / \text { science. } 1167747}$

9. Moore, J.E., The birth of topological insulators, Nature, 2010, vol. 464, no. 7286, p. 194.

10. Hsieh, D., Xia, Y., Quian, D., Wray, L., et al., A tunable topological insulator in the spin helical Dirac transport regime, Nature, 2009, vol. 460, no. 7257, p. 1101. https://doi.org/10.1038/nature08234

11. Tang, S. and Dresselhaus, M.S., Constructing anisotropic Single-Dirac-Cones in $\mathrm{Bi}_{1-\mathrm{x}} \mathrm{Sb}_{\mathrm{x}}$ thin films, Nano Letters, 2012, vol. 12, no. 4, p. 2021.

12. Brandt, N.B., Gitsu, D.V., Nikolaeva, A.A. and Ponomarev, Ya.G., Investigation of size effects in thin cylindrical bismuth single crystals in a magnetic field, JETP, 1977, vol. 45, no. 6, p. 1226.

13. Nikolaeva, A., Huber, T.E., Gitsu, D. and Konopko, L., Diameter-dependent thermopower of bismuth nanowires, Phys. Rew. B., 2008, vol. 77, no. 3, art. 035422 . https://doi.org/10.1103/PhysRevB.77.035422

14. Konopko, L.A., Nikolaeva, A.A., Huber, T.E. and Ansermet, J.-P., Surface states transport in topological insulator $\mathrm{Bi}_{0.83} \mathrm{Sb}_{0.17}$ nanowires, J. Low Temp Phys., 2016, vol. 185, no. 5, p. 673.

15. Konopko, L., Nikolaeva, A., Huber, T.E., and Rogacki, K., Quantum oscillations in nanowires of topological insulator Bi0.83Sb0.17, Appl. Surf. Sci., 2020, vol. 526, art. ID: 146750. https://doi.org/10.1016/j.apsusc.2020.146750

16. Nikolaeva, A.A., Konopko, L.A., Huber, T.E., Ansermet, J.-Ph., et al., Thermoelectric phenomena in $\mathrm{Bi}_{1-\mathrm{x}} \mathrm{Sb}_{\mathrm{x}}$ nanowires in semimetal and gapless region, J. Nanoelectron. Optoelectron, 2012, vol. 7, no. 7, p. 671.

17. Taskin, A.A., Segawa, K. and Ando Y., Oscillatory angular dependence of the magnetoresistance in a topological insulator oscillations in a topological insulator $\mathrm{Bi}_{1-\mathrm{x}} \mathrm{Sb}_{\mathrm{x}}$, Phys. Rev. B, 2010, vol. 82, no. 12, art. $\quad$ ID: $\quad 121302$

doi: 10.1103/PhysRevB.82.121302

(R).

18. Редько, Н.А., Каган, И.Д., Волков, Н.П., Электронный топологический переход в области магнитных полей квантового предела при НII С2 в полупроводниковом сплаве n-Bi-Sb, ЖЭТФ, 2010, т. 138, № 2(8), с. 271.

19. Taskin, A.A. and Ando, Y., Quantum oscillations in a topological insulator $\mathrm{Bi}_{1-\mathrm{x}} \mathrm{Sb}_{\mathrm{x}}$, Phys. Rev. B., 2009, vol. 80, no. 8, art. ID: 085303. doi: 10.1103/PhysRevB.80.085303.

20. Qu, D.-X., Hor, Y.S., Xiong, J., Cava, R.J., et al., Quantum oscillations and Hall anomaly of surface states in the topological insulator $\mathrm{Bi}_{2} \mathrm{Te}_{3}$, Science, 2010, vol. 329, no. 5993, p. 821.

21. Shoenberg, D., Magnetic oscillations in metals, Ser. Monographs on Physics, Cambridge University Cambridge, 2009, 596 p.

\section{Summary}

The transport properties, magnetoresistance, and Shubnikov-de Haas (SdH) oscillations of glass-coated $\mathrm{Bi}_{0.92} \mathrm{Sb}_{0.08}$ single-crystal wires with diameters of $180 \mathrm{~nm}$ to $2.2 \mu \mathrm{m}$ and the (1011) orientation along the wire axis, which are prepared by liquid phase casting, have been studied. For the first time, it has been found that the energy gap $\Delta E$ increases by a factor of 4 with a decrease in the wire diameter $d$ owing to the manifestation of the quantum size effect. This significant increase in the energy gap can occur under conditions of an energymomentum linear dispersion relation, which is characteristic of both the gapless state and the surface states of a topological insulator. It has been shown that, in a strong magnetic field at low temperatures, a semiconductor-semimetal transition occurs; it is evident in the temperature dependences of resistance in a magnetic field. An analysis of the SdH oscillations, namely, the phase shift of the Landau levels and the features of the angular dependences of the oscillation periods, suggests that the combination of the manifestation of the topological insulator properties and the quantum size effect leads to the occurrence of new effects in low-dimensional structures, which requires new scientific approaches and applications in microelectronics

Keywords: quantum size effect, topological insulator, surface state, single-crystal nanowires, quantum oscillations, transverse magnetoresistance 